\title{
High-Precision NBPP Timing Measurements at Nançay
}

\section{Cognard}

CNRS-LPCE, 45071 Orléans FRANCE email: icognard@cnrs-orleans.fr

J.-F. Lestrade

Observatoire de Paris-Meudon 92195 Meudon FRANCE

D.C. Backer

\author{
Astronomy Dept, University of California, Berkeley, CA 94270, USA \\ P.S. Ray, R.S. Foster, B.J. Cadwell
}

Code 7213, naval Research Laboratory, Washington, DC 20375, USA

The Nançay radiotelescope in France is a large collecting area $\left(7000 \mathrm{~m}^{2}\right)$ with receivers around $1.4,1.7$ and $3.5 \mathrm{GHz}$. At this observatory, we are conducting frequent high-precision timing observations of 5 millisecond pulsars (PSR $\mathrm{B} 1937+21, \mathrm{~B} 1821-24$ since 1988 ; J1643-1224, J1713+0747 and B1620-26 since 1996) with a swept frequency oscilllator based on a DDS as a dedisperser.

The most interesting result from these dense series of observations is the detection of several Extreme Scattering Events in direction of B1937+21 and possibly in direction of B1821-24 (Cognard, 1993, Nature, 366, 320 ; Cognard \& Lestrade, 1996, in ASP Conf. Ser. Vol 105, Pulsars : Problems and Progress (San Francisco: ASP), 469 ; Lestrade, Rickett \& Cognard 1998, A \&A, 334, 1068). The flux density variations and TOA fluctuations observed have been used to estimate the size (several AU) and electron density (a few hundreds electrons $\mathrm{cm}^{-3}$ ) of the discrete ionised clouds localized in the interstellar medium that are thought to be responsible for this phenomena. The number of events recorded at Nançay in direction of $\mathrm{B} 1937+21$ yields the space density $10^{5}-10^{6}$ $\mathrm{pc}^{-3}$ for these clouds. This density is very large and is about 100 times higher than the density estimated from Extreme Scattering Events observed in direction of extragalactic radio sources (Fiedler et al., 1994, $A p J, 430,581$ ). This might mean that the line of sight to B1937+21 is peculiar. This is being investigated with additional observations of a larger array of pulsars at Nançay with the Navy-Berkeley-Pulsar Processor (Figure 1) This proccssor NBPP (Foster et al., in ASP Conf. Ser. Vol 105, Pulsars : Problems and Progress (San Francisco: ASP), 25) has been used to acquire pulsar search data for 2 years at Nancay and we are now using its real-time folding capability.

We have selected $\sim 20$ millisecond pulsars with period time derivatives smaller than $\dot{P}$ of the most rotationally-stable pulsar B1855+09 (i.e. $\leq 2 \times$ $10^{-20} s / s$ ). These observations should also be useful to constrain the stochastic background of gravitational waves. 


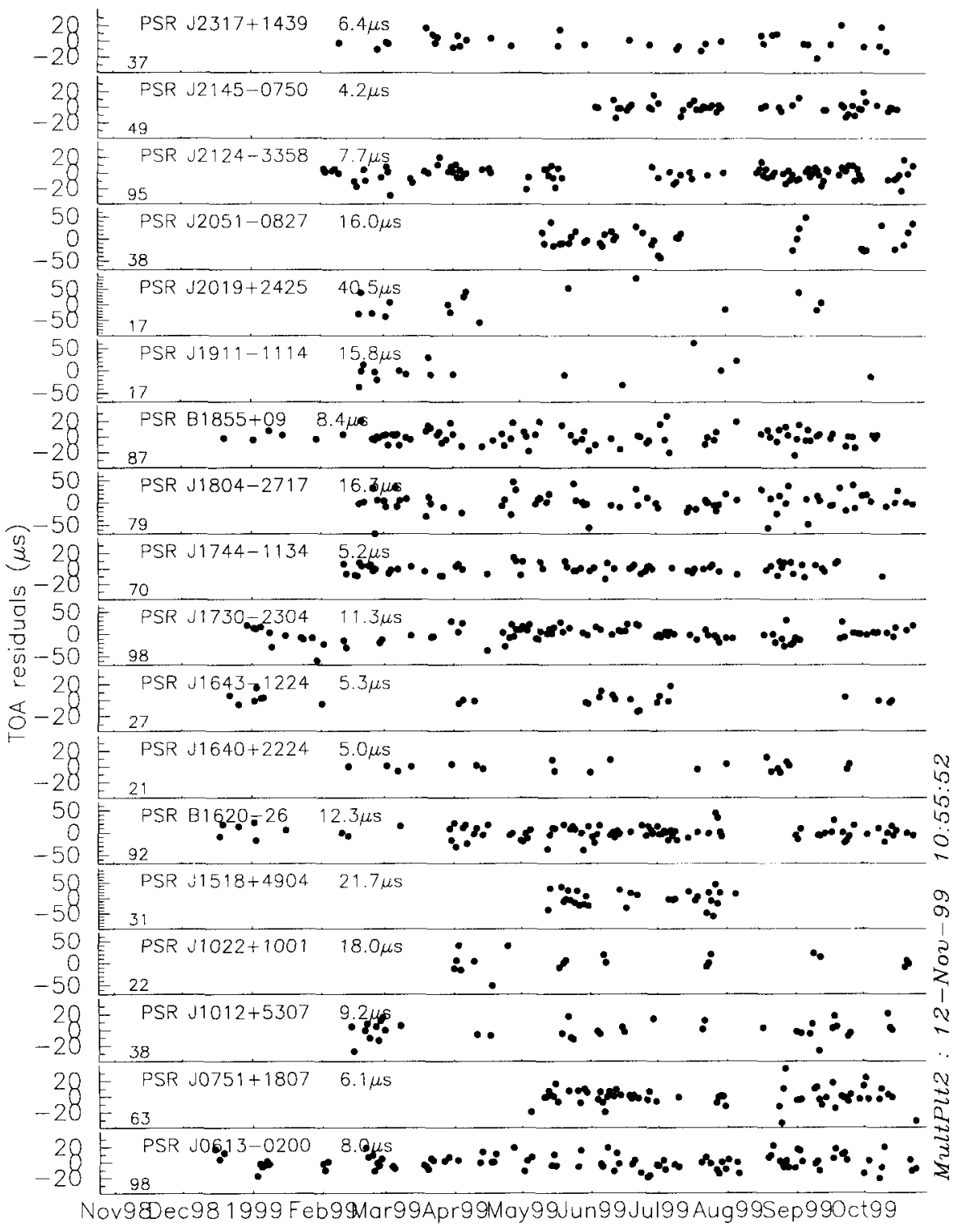

Figure 1. Time of arrivals residuals for all the newly observed millisecond pulsars at Nançay with the NBPP. TOA residuals rms and number of observations are quoted. 